\title{
Development of a Video Network for Efficient Dissemination of the Graphical Images in a Collaborative Environment.
}

\author{
Anatoliy Gordonov \& Michael Kress \\ gordonov@postbox.csi.cuny.edu kress@postbox.csi.cuny.edu \\ The College of Staten Island/City University of New York (USA)
}

\begin{abstract}
Video distribution inside a local area network can impede or even paralyze normal data transmission activities. The problem can be solved, at least for a while, by compression and by increasing bandwidth, but that solution can become excessively costly or otherwise impractical. Moreover, experience indicates that usage quickly expands to test the limits of bandwidth. In this paper we introduce and analyze the architecture of a Hybrid Analog Digital Video Network (ADViNet) which separates video distribution from standard data handling functions. The network preserves the features of a standard digital network and, in addition, provides efficient real-time full-screen video transmission through a separate analog communication medium. A specially developed control and management protocol is discussed. For all practical purposes ADViNet may be used when graphical images have to be distributed among many nodes of a local area network. It relieves the burden of video distribution and allows users to combine efficient video data transmission with normal regular network activities.
\end{abstract}

Keywords: Hybrid AnaloglDigital Video Network, real-time full-screen video transmission, $\quad$ collaborative environment.

\section{Introduction}

\section{The Problem Statement}

Today's desktop video is an emerging technology. Business, industrial, and educational systems can all benefit from presenting video images to many users simultaneously.

Video transmission consumes a great deal of network resources. A simple calculation shows (Goldman, J., 1995) that uncompressed full-screen motion video requires more than 80-megabits/second bandwidth. The majority of local area networks (LAN), which have the transmission speed of 10-16 megabits/second, cannot carry such traffic. New developments (Fast Ethernet, 100VG-AnyLAN) are not yet widespread. Although they can improve the performance of video distribution, uncompressed video traffic is unacceptable even for these networks.

Material published as part of this journal, either on-line or in print, is copyrighted by the publisher of Informing Science. Permission to make digital or paper copy of part or all of these works for personal or classroom use is granted without fee provided that the copies are not made or distributed for profit or commercial advantage AND that copies 1) bear this notice in full and 2) give the full citation on the first page. It is permissible to abstract these works so long as credit is given. To copy in all other cases or to republish or to post on a server or to redistribute to lists requires specific permission and payment of a fee. Contact Editor@gise.org to request redistribution

permission.
The main directions in reducing bandwidth requirements are:

- employing various video compression techniques. By using an appropriate compression technique, the transmission of a high resolution compressed video may require 6-24 megabits/second bandwidth;

- reducing the amount of video data, which has to be transmitted. It can be done by reducing the number of pixels per line, lines per frame, and frames per second. Of course, this reduction may be done at the expense of video frame resolution and full motion video quality. As an example, we can mention the CCITT's ( International Telegraph and Telephone Consultive Committee (now ITU-T) H.261 video transmission standard. It defines two different video resolution formats: Common Interface Format with 352 pixels by 288 lines per frame and Quarter Common Interface Format with 176 pixels by 144 lines per frame.

The delay-sensitive nature of video information introduces another problem of video transmission. Ethernet, Token Ring, and FDDI networks allow delays in transmission of video data. One of the ways of solving this problem is employing such services as frame relay and ATM which are not widely used yet.

Any video transmission in a local area network must coex- 


\section{Development of a Video Network}

ist with other network activities. A huge amount of data involved in a video transmission slows down all other local area network operations. That can be very frustrating for users who are not involved in the current video session. In a real-time system the situation may even become dangerous because of delays in emergency information.

Unfortunately, none of the above mentioned strategies can solve all the problems of video distribution. All of them have their own benefits and drawbacks.

In this paper we consider one possible way of solving the problem of video distribution. We examine a Hybrid Analog/Digital Video Network (ADViNet) which uses two parallel communication media: analog - for transmitting video images, digital - for data transmission. We present the model and architecture of a video network and ADViNet protocol. We also analyze the benefits and weaknesses of the video networks.

\section{Background}

The typical physical architecture of a Hybrid Ana-

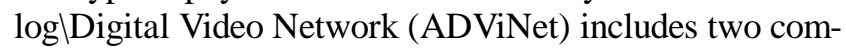
munication media: digital and analog. The digital medium is used by a regular digital network according to its type and topology. The analog medium combines a VGA bus with several additional control lines. All the monitors in the LAN are connected to this bus by special switches. Each monitor can work in one of the two states: local when the user can work independently with his/her workstation; and remote - when the monitor is connected to a remote source of video information. The analog bus is controlled centrally from the control panel located near the master station.

We have implemented ADViNet in a classroom/laboratory at the College of Staten Island. On the physical level in our video network environment we use a Computer Video Web (ComWeb). ComWeb is a commercially available pure hardware system for video distribution ("Computer Video Web", 1996). The analog transmission medium has a centralized manual control on the physical level at the control panel connected to the main hub or control can be programmed from the central station. It features a limited number of operations.

In this architecture video information is very easily transmitted between a source and destination using the analog bus. The video transmission can be done concurrently with regular digital network operations. The dedicated communication medium for video distribution provides fast full-screen real-time video communication. It does not introduce data transmission delays in the digital part of the network.

We now describe the major steps of ADViNet development. The main goals of this development are:

- to develop a decentralized model of the ADViNet architecture that will provide an opportunity of a programming control of the video network from any station;

- to enhance flexibility of the video network by increasing the number of operations that can be implemented from a workstation;

- to introduce new operations including intelligent ones.

In order to achieve the goals mentioned above, we are going to introduce new logical architecture and a model of a video network and develop a multilayer control and management protocol.

\section{Model and Architecture of a Video Network}

In this section we present the generalized architecture of ADViNet. It demonstrates how the functionality and flexibility of a video network may be extended beyond the boundaries of pure hardware systems.

We distinguish two types of video sessions. The first type, Individual Video Session (IVS), is established between a single user and a video server or between two users. Many such sessions may be established simultaneously, and they may have different hardware and software requirements. The second type of video sessions, Public Video Sessions (PVS), is established among many users simultaneously, all participating in the same activity.

In general, digital networks, regardless of type and topology, can be represented as a set of nodes interconnected through a communication medium. Each node constitutes a separate computer with the necessary external devices (such as display, keyboard, mouse, etc.) locally connected to the main processor unit. Neither of these devices works independently from the local node. Communication in the network consists of data transmission between processor units. This traditional network architecture is inefficient for transmitting video information. For the local area network in which PVSs are used frequently, another network model is much more efficient.

Let us consider two sets of network nodes: 
- $\mathrm{P}=\{\mathrm{P} 1, \mathrm{P} 2, \ldots, \mathrm{Pn}\}$ is a set of processor units. These are intelligent nodes which can produce images;

- $\mathrm{D}=\{\mathrm{D} 1, \mathrm{D} 2, \ldots, \mathrm{Dm}\}$ is a set of display units. These Di can display images produced by any processor unit.

We limit our consideration to these two groups of nodes. Although other groups of external devices (such as keyboards, mice etc.) may be included in a network, we do not consider them here. In the traditional network architecture $\mathrm{n}=\mathrm{m}$, all Pi are connected to the network and any Di is connected to the appropriate $\mathrm{Pi}$. In the architecture, which we consider, $\mathrm{n}$ is not necessarily equal to $\mathrm{m}$ and processor and display units may be interconnected in several different ways. One processor unit $(\mathrm{Pi})$ may be connected to several display units. Images produced by Pi may be broadcast to all the elements of the set D or multicast to a subset of D.

This occurs during PVS when several users need to observe images supplied by one processor unit. The opposite situation occurs when several processor units generate images that could be observed in turn by one display element. The number of processor units may vary from an entire set $\mathrm{P}$ to a subset of $\mathrm{P}$. It is important to note that all the connections in ADViNet are not permanent. The number of interconnected nodes and the type of connections may be changed dynamically at any time. This makes ADViNet flexible and allows us to introduce new network operations and new types of connections that require multiple, dynamic, real-time network reconfiguration.

In general, the proposed ADViNet architecture follows these basic principles:

1. A special communication medium is used to ensure high-speed transmission of video information;

2. The additional medium transmits graphical data in an analog form which guarantees high-speed video distribution in a local area network for many users simultaneously;

3. The analog medium does not substitute for a standard digital network medium. Moreover, both media work concurrently and independently on the physical level;
4. ADViNet has decentralized control that allows any processor unit to provide network control by issuing appropriate commands.

The hardware architecture of ADViNet is shown in Figure 1. Any Di is connected to Pi through a special remotely controlled switch. By default, all the displays are connected to their processor unit locally. In case of necessity, a display or processor unit may be switched to an analog communication medium to participate in a PVS. The digital medium is used by a regular digital network according to its type and topology. The analog medium is dedicated to video distribution. It combines a VGA bus with several additional control lines. Any processor unit (as well as VCR, video camera, laser disk, and others) may serve as a source of video information for a session. Because an ana$\log$ bus is connected to a processor unit through a standard video card, a processor unit works with an analog bus in the same way that it works with a local display. We extend the characteristics and flexibility of a video network by introducing a new logical structure for the network and imple-
Figure 1. Physical Architecture of the Hybrid Analog/Digital Video Network

Analog Medium

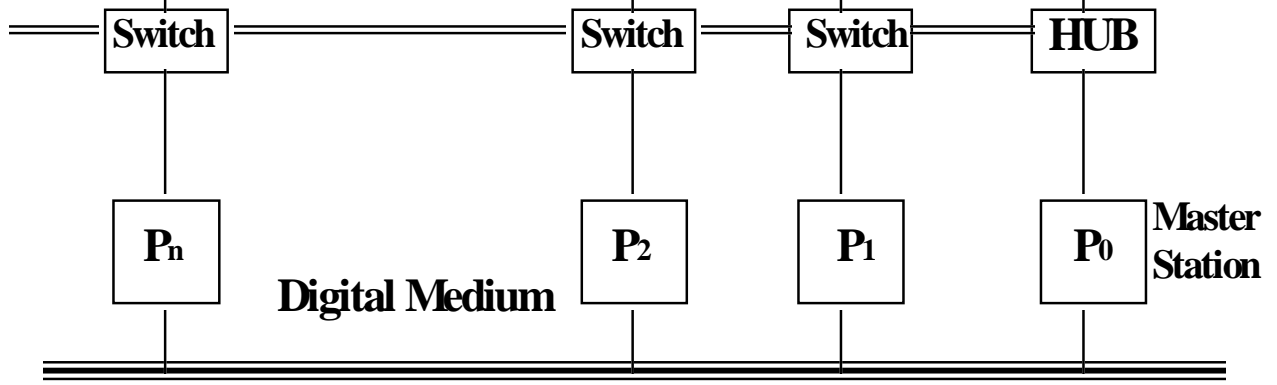

menting a programming control component. The control and management operations of the video network are supported through a specially developed multilayer communication protocol.

\section{ADViNet Protocol}

The ADViNet protocol includes two groups of operations: control operations and management operations. The first group covers the operations that directly affect the distribution of video information through the analog medium. Users participating in the video session may employ these operations (we call them controls) to control video transmission. Some of the controls may be issued only from the master station, others-from any workstation. 


\section{Development of a Video Network}

Management operations include network administration commands. Most of them can be used only by the network administrator and issued only from the master station.

Two main ideas underlie the ADViNet development :

- concurrent use of an analog communication medium for video distribution and a digital medium for the control;

- conversion of a pure rigid hardware video distribution system with centralized control into a decentralized flexible and programmable video network.

Both of these ideas have a major impact on the structure and content of the ADViNet control and management protocol.

The structure of the ADViNet protocol is shown in Figure 2. The protocol includes two layers: physical and logical. Physical controls are issued from the master station that transmits them directly to the network control hardware. Due to the hardware architecture of ADViNet, no physical layer controls can be sent directly from any workstation. Instead, the logical layer includes similar controls that can be sent from a workstation to the master station. Upon receiving these controls, the master station forwards them to the network control hardware through the physical layer. Logical controls can be sent from either the master station or any workstation. The execution of a logical operation requires negotiation between the master station and a workstation. The complexity of this negotiation may vary for different controls.

The logical layer incorporates one sublayer, which we call an intelligent logical sublayer. The intelligent logical sublayer allows us to implement conditional communication operations when the next logical layer control is chosen as a result of certain logical operations. These logical operations are executed during the communication and embedded into the protocol. A control operation may be derived as a result of the numerical and/or logical calculation. The execution of such a control operation may take place immediately or it may be delayed for some time if required resources are not available. The control operation by itself may be complex and include several simple controls. We call these control operations intelligent controls and the network which supports them an intelligent network (Ahamed, S., Lawrence, V., 1997).

Some controls depend on the parameters of the network environment. The user cannot change these parameters. Only the network administrator can do so using the ADViNet management protocol. Among these parameters are
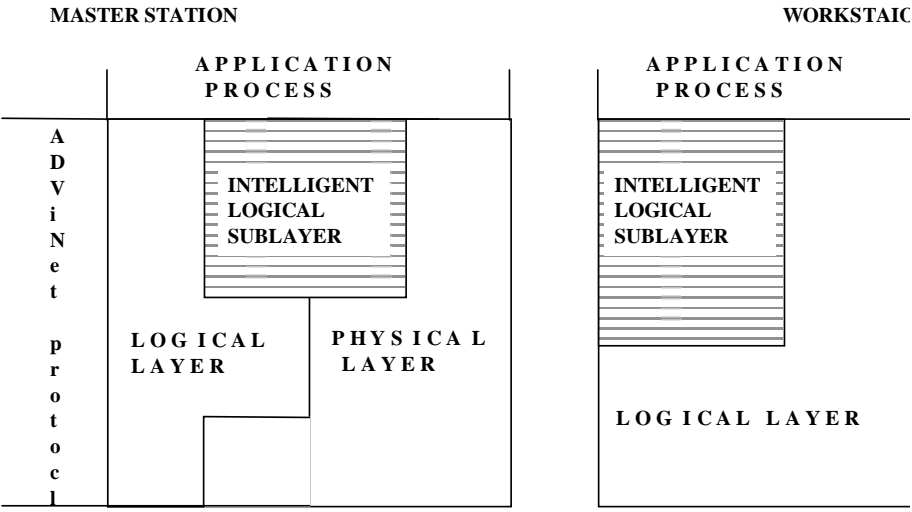

Figure 2. Structure of the ADViNet Protocol

workstation registration, workstation priority, setting or changing video session password and session time limit, etc. Any part of the protocol is directly accessible to a user. An application may access ADViNet through the intelligent logical sublayer, logical layer or physical layer. From the master station, a user may issue:

- a physical control that is directed to the main hub and will make the required switches;

- a logical layer control that supports network management or communication with a workstation;

- an intelligent logical sublayer control that allows users to provide complex operations by combining several logical and physical controls together with data analysis and decision making elements.

From a workstation, a user can send:

- a logical layer control that starts a distributed communication procedure between the workstation and the master station. The procedure is partially executed on the master station and partially on the workstation. As a result of this procedure, a set of the physical controls is formed and executed on the master station;

- an intelligent logical sublayer control that initiates the appropriate intelligent network function on the master station.

Let us consider the main ADViNet control and management operations. From the point of view of video distribution, there are two main operations. The first operation (we call it "One-to-Many") is designed to transmit images from one network node to several displays. An image may be sent to all network nodes ("Broadcast") or to some of them ("Multicast"). The broadcast and multicast operations may 


\section{Gordonov \& Kress}

be executed on both master and workstations. The physical layer of the ADViNet protocol is employed to perform these operations on the master station. Any workstation uses the logical layer of the protocol to issue a broadcast or multicast control. According to the protocol, a workstation (we call it a primary station ) must send a request to the master station asking permission to provide "One-toMany" video operation. If currently there is no active session and resources are available, the master station sends a confirmation back to the workstation and executes all the necessary steps through the physical layer of the protocol. If there is an active session and the current request (node) has lower or equal priority compared to the current primary station, the request of the workstation is rejected.

A multicast operation demands an additional set of parameters - the identifying numbers of the stations participating in a session. A user submits these numbers after the confirmation has been received.

As we have discussed before, if the video network is currently busy with another operation, a request may be declined. However, if the current operation is a conditional broadcast, such refusals can be avoided. In this case, an intelligent logical sublayer is employed. During the conditional broadcast operation, the master station periodically sends requests to all the workstations asking them if a broadcast operation is needed. After receiving answers from all the workstations, the master station forms a request queue. The requests are placed into the queue according to the queue type ( "first come - first served", priorities, etc.). The advance from one queue element to another may be done automatically by allowing each workstation to broadcast for a particular interval of time or manually from the master station. The actions mentioned above are covered by the three protocol states: Real-Time Data Acquisition state, Queue Management state, and Broadcast state. These three states are executed in a loop until the node that initiated the intelligent broadcast operation sends a request to stop it. Figure 3 illustrates the state transition diagram of the ADViNet protocol for the intelligent broadcast and explains the switches from one state to another.

The conditional multicast function, in some sense, is similar to the conditional broadcast in that, under certain circumstances, an image is sent from the source node to a group of destination nodes. Apart from this similarity, however, conditional multicasting is a more complicated operation and incorporates four different functions.

The second video control operation that we consider is "Many-to-One". During this operation, the images are transmitted consecutively (with a delay) from all the work- stations participating in the operation to the primary node that initiated the operation. Any workstation or the master station may play the role of the primary node. If the master station initiated the operation, only the physical layer of the ADViNet protocol can be employed. The master station may provide network monitoring if all the nodes of ADViNet are involved or group monitoring if only a subset of the nodes participates in the operation. If a workstation is used as a primary node, the protocol includes logical communications between the workstation and the master station. During the communications, the primary workstation needs to receive permission from the master station to provide monitoring. The permission may not be issued if the network is busy and the current primary node has higher priority than the node requesting the operation. The request may also be rejected if the initiating node is not allowed to execute the operation. The request for network monitoring initiates a procedure on the master station that consecutively transmits images from the network nodes to the primary station. A sequence of the physical layer controls is used for this purpose. A group monitoring control allows the primary station to monitor a subset of the network nodes. The primary node assigns the set of participating stations when the operation is requested.

The intelligent logical sublayer of the ADViNet protocol contains an adaptive group monitoring control. This control is similar to group monitoring except that the size of the group may vary during the operation. Participation in the group is defined for any node by calculating the state function of the node. (Gordonov, A., 1997). This function depends on real-time variables and is recalculated periodically. The variables are bound to the external events. The state function together with the external variables provides a connection between the external world and the state of the node. The state function itself is Boolean. If the value of the function is true, the node is included in the group of monitored nodes, otherwise, the node is excluded from the group. Adaptive group monitoring may be initiated from both the master station and any workstation. In the first case, the following steps are implemented:

- Real-time data acquisition about the states of the nodes participating in the operation;

- State analysis and formation of a set of nodes with the state function equal to true;

- Calculation of a monitoring time for a single group. During this interval, all the members of the group are monitored one time. The time is calculated as the product of the scan rate and the number of elements in the group; 


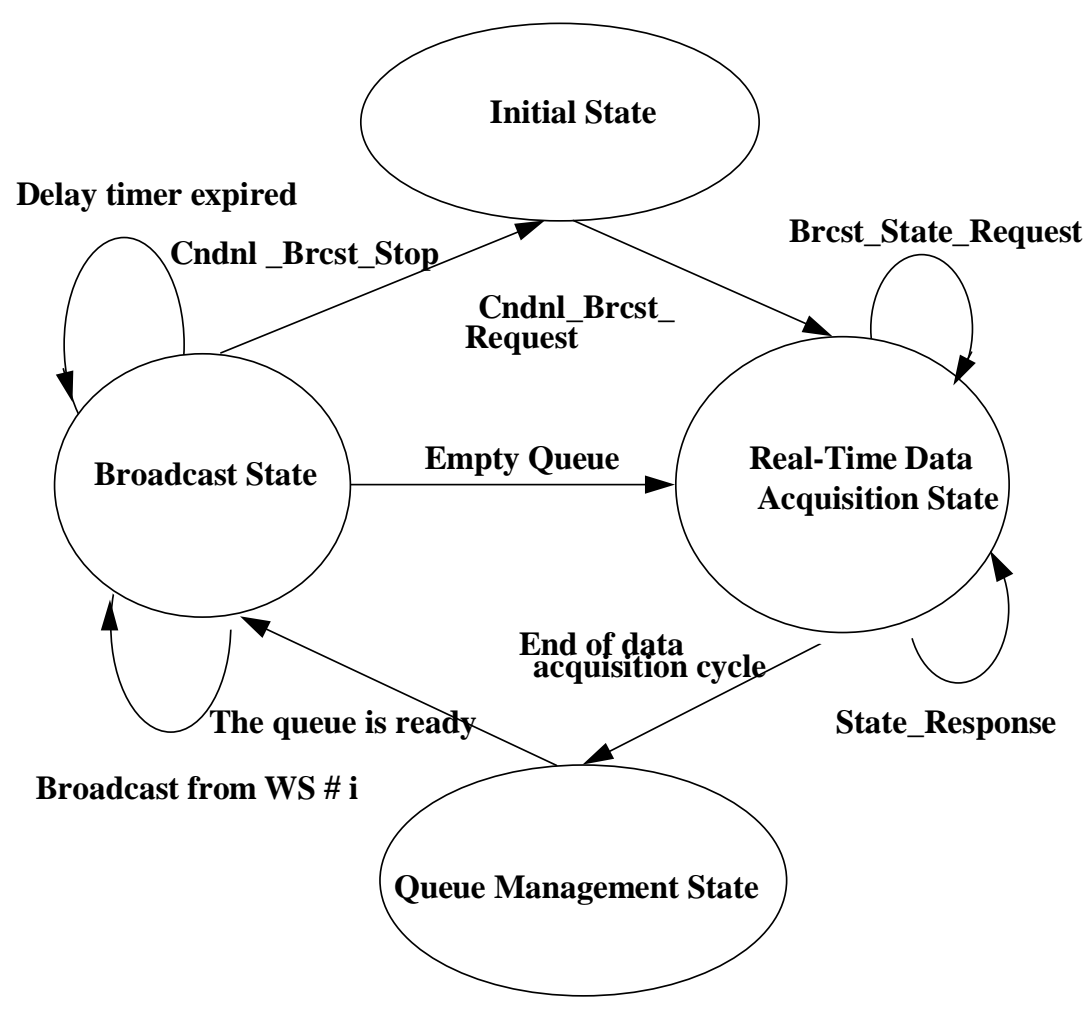

Figure 3. State Transition Diagram of the ADViNet Protocol for Conditional Broadcast.

- Reconfiguration of ADViNet for monitoring the new set of nodes. Start the monitoring operation. For this step, a physical layer protocol is employed. Set the expiration timer;

- When the timer expires, interrupt the physical monitoring operation;

- If there is no request to stop the monitoring operation, go to the real-time data acquisition step.

Adaptive monitoring from a workstation requires a preliminary negotiation between the master station and the workstation. The following steps are involved in the execution of the adaptive monitoring operation from a workstation:

- a workstation sends a request to the master station asking permission to execute the adaptive group monitoring operation;

- the master station permits the operation if the network is not currently busy, and the workstation is allowed to execute the monitoring operation; help.
- the workstation submits to the master station a set of the nodes participating in the operation;

- the master station provides real-time data acquisition about the states of the nodes participating in the operation, analyzes the states, and forms a set of nodes with the state function equal to true;

- the master station consecutively transmits images from the nodes from the group to the workstation which initiated the operation. The physical layer control is used. Monitoring time for each node is defined by the scan rate;

- after the nodes from the group are monitored, the master station goes back to the real-time data acquisition step;

- the operation stops after the workstation which initiated it issues the appropriate request or a node with higher priority interrupts the current operation.

The adaptive group monitoring control allows users to observe and supervise only those nodes in the network that satisfy a certain criterion. The criterion is embedded into the state functions. If a state function is true, we make a decision that the criterion for a node is satisfied and the node must be included into the monitored group.

As an example, we can consider an adaptive group monitoring in a classroom that provides real-time help to students who have difficulties. For this purpose, the master station gathers and analyzes the information about the current state of any student's station. This information shows each student's performance (compared to the preassigned schedule). Based on the analysis, the master station dynamically changes the configuration of ADViNet: new nodes can be added to the monitored group, and others can be removed. The instructor can always observe only those students who are behind schedule and give them real-time

So far, we have considered ADViNet control operations. These operations deal with controlling an analog medium. There are several more groups of protocol functions having the main goal of supporting control operations. We unite 
them under the name of management functions. All management functions are executed at the logical layer of the ADViNet protocol. No physical layer controls are associated with management functions.

There are several categories of the management functions:

- $\quad$ session management;

- accounting management;

- $\quad$ security management;

- fault management.

In the next two sections we answer the questions why and where we need to use ADViNet.

\section{ADViNet Analysis}

Now we analyze the main strengths and weaknesses of the ADViNet architecture proposed in this paper. We compare the ADViNet features with the functions of pure hardware video networks as well as regular local area networks.

Compared to pure hardware systems (such as ComWeb), ADViNet has the following advantages:

- decentralized logical structure that allows any station to control the network;

- better functionality in network control and management based on a specially developed protocol;

- $\quad$ greater flexibility through a logical structure using a new protocol that allows users to create new video network operations;

- $\quad$ support for intelligent video network operations.

ADViNet covers all the operations of its predecessor (ComWeb), but the only weakness should be mentioned: ADViNet is no longer a pure hardware system and includes a software component. This component depends on the specific computer architecture and operating system environment. The problem may be overcome by creating several alternatives in the software.

Let us compare video distribution in digital local area networks with and without ADViNet. We can find the following benefits of using ADViNet in a local area network:
- does not slow down regular LAN activities;

- has special functions for collaborative work (such as multicasting and monitoring);

- supports the creation and function of the intelligent network operations that may considerably enhance the ways of how the video information can be used;

- does not consume LAN resources;

- does not require changes in the existing LAN environment;

- has simple installation as an add-on to any LAN.

On the other hand, there are several weaknesses of ADViNet that narrow its usage in some applications. First of all, the number of concurrent sessions in the ADViNet environment is very limited. This number depends on the architecture of the analog medium: it is possible to open a separate video session for each separate analog bus.

Usually, up to 4 analog buses may be connected to the main hub. Nevertheless, several groups of applications require only one open video session at a time. ADViNet supports these applications.

The second disadvantage is that the installation of ADViNet requires additional wiring and equipment (main hub, switches). Although the cables and additional hardware are not expensive (and become cheaper every day) and the installation itself is simple, some extra money and work are required.

The analysis shows that ADViNet has several important features that make it very useful in the collaborative environments where video information is to be transmitted to many users simultaneously during one video session. This session may coexist with other network operations and does not introduce any delay for them. The cost of a video network mostly constitutes of the cost of hardware and installation and depends on the number of stations and their distribution in the local area network. In some applications ADViNet may substitute for videoconferencing systems that makes its use more cost effective ( especially if we consider all the extra features of ADViNet). In other applications $\mathrm{ADViNet}$ may be used as an addition to regular videoconferencing environments, but the benefits mentioned above are worth the extra cost. Below we are going to consider several examples of such applications.

- supports full-screen motion video distribution regardless of the LAN type and topology; 


\section{Practical Consideration}

AnaloglDigital Video Networks are relatively new on the market. They appeared several years ago ("Computer Video Web", "V-Net") and have similar physical architecture and functions. They have already found their place in electronic classroom environments. Video helps an instructor solve problems more easily by illustrating computer-based methods of solution and making them more understandable. ADViNet presented in this paper offers much better flexibility in terms of network control and allows the instructor to use intelligent video network operations in class monitoring. This is a step toward creating a richer and more efficient teaching environment.

At the College of Staten Island/City University of New York we have three computer laboratories equipped with video networks. The faculty is using video networks for various kinds of classroom activities that require the delivery of computer-mediated information to the students or for instructor assistance during the laboratory works, drills, exercises, and tests. In a video network environment we teach computer science courses of various levels: computer literacy courses, Assembly language, Data Structures, Software Engineering, and others. Our faculty experience shows that Hybrid Analog Digital Networks prove to be useful in the classroom environment. Not only do they support major instructional activities, but they are also very efficient in the distribution of full-screen motion video and other graphical images. ADViNet does not introduce extra traffic in the local area network and does not slow down other network operations. The use of a video network is an efficient teaching tool in those courses where the course content requires an extensive use of lecturing and presentations from various visual sources. In advanced classes, laboratory works and seminars, video networks have proven productive for modeling, individual and group tutoring, and monitoring. Student surveys suggest that the students like to take classes in a classroom equipped with a video network (Gordonov, A., Kress, M., Carlin, M., 1997). They prefer video networks to other systems of video distribution (such as big screens) and consider as an effective teaching tool. More details about using video networks in educational systems may be found in ( Gordonov A., Kress M., Klibaner R.,1998, and Gordonov, A., Kress, M., 1997).

Distance learning environments may serve as another example of the ADViNet application. In this case, ADViNet takes care of the local video distribution while one of the standard videoconferencing systems (such as Intel ProShare ${ }^{\mathrm{TM}}$, Tenberg ${ }^{\circledR}$ or PictureTel ${ }^{\circledR}$ ) is responsible for transmitting video to remote sites. The combination of these two technologies permits to use the ADViNet benefits in multisite learning environments. Many colleges already have several local classrooms equipped with the video net- works and try to find a way of their connection. The systems with this distributed architecture do not exist right now. The first version of the software for the remote control of the ADViNet site (which is being developed by ComWeb Technology Group, Inc. with the authors' participation) will be available next year.

The applications of ADViNet are not limited to educational systems. Video networks are used for network management in the local area networks. By employing a video network, an administrator can provide management and control functions virtually from any workstation including software installation and changing network parameters.

Another class of applications that can benefit from using ADViNet is industrial real-time systems. Video transmission in real-time systems allows operators to enhance the quality and efficiency of the control operations. Real-time systems usually use a regular local area network for data acquisition and distribution. At least a considerable part of this information is time-sensitive and cannot tolerate delays. It makes most of the existing technologies of video transmission unacceptable in real-time environments. ADViNet can not only remedy these deficiencies but also cover the main requirements for video distribution operations in industrial control systems. It supports a collaboration of a group of specialists in resolving emergency situations (the case where regular videoconferencing systems cannot be used because of the considerable delays introduced in the network). The application of the intelligent ADViNet operations (such as monitoring and multicasting) allows the operators to concentrate on the development and analysis of a particular emergency situation and helps them find a better decision within a shorter period of time. The problems of incorporating ADViNet in real-time systems are being developed by the authors in collaboration with AtlanticTransGas Systems, Ltd., a major software developer and hardware integrator for real-time control systems for the gas transportation companies in Siberia (Russia).

\section{Conclusion}

In this paper we introduced and analyzed the architecture of a local area network, which separates video distribution from standard data handling functions. The network preserves the features of a standard digital network and, in addition, provides efficient real-time full-screen video transmission through a separate analog communication medium. A specially developed control and management protocol was discussed. For all practical purposes ADViNet may be used when graphical images have to be distributed among many nodes of a local area network. It relieves the burden of video distribution and allows users to combine efficient video data transmission with normal regular network activities. 
The major contributions of this paper are the development of the:

- general concept of a video network;

- decentralized model and logical architecture of a video network;

- concept and implementation of intelligent adaptive video operations;

- multilayer ADViNet control and management protocol;

- examples of the ADViNet applications and directions of the future development.

\section{References}

"Computer Video Web", ComWeb Technology Group, Inc. Manual, Wayne, NJ, 1996

Gordonov, A., Kress, M., Blank, A., Moroh, M., "ComWeb: An Electronic Classroom for Teaching Computer Literacy", Computers and Education. An International Journal. Vol. 29, No.4, pp181-187, 1997
Ahamed, S. \& Lawrence, V. Intelligent Broadband Multimedia Networks, Boston: KAP, 1997, p.5.

Gordonov A.: "Analog/Digital Networks for Real-Time Video Distribution with Extra Computational Capabilities.” REAL-TIME IMAGING '97 Conference, San Jose, California, 1997, pp172-179.

Gordonov, A., Kress, M., Carlin, M., "Video Networks in the Electronic Classroom Environment and Analysis of Student Preferences in the Development of a Network-Based Video Distribution System", 1997 ASCUE Summer Conference, Myrtle Beach, South Carolina.

James E. Goldman, “Applied Data Communication. A Business-oriented Approach”, WILEY, NY, 1995, p. 504.

“V-Net", Interactive Training System ,INLINE, Inc., La Habra, CA, 1996

Gordonov A., Kress M., Klibaner R., "Development of a Real-Time Intelligent Network Environment ",Proceedings of the 1998 Association of Small Computer Users in Education (ASCUE) Summer Conference, Myrtle Beach, South, Carolina, June 7-11, 1998.

Gordonov, A., Kress, M., "Educational Analog/Digital Video Networks", AACE ED-TELECOM Conference, pp. 1222-1223, Calgary, Canada, June 1997. 